\title{
Factores contextuales relacionados a las conductas de autolesión no suicidas: una revisión sistemática
}

\section{Contextual factors related to non-suicidal self-harm behaviors: A systematic review}

\author{
Karen Guadalupe Duarte Tánori, José Ángel Vera Noriega \\ y Daniel Fregoso Borrego ${ }^{1}$
}

Citación: Duarte Tánori, K.G., Vera Noriega, J.Á. y Fregoso Borrego, D. (2021). Factores contextuales relacionados a las conductas de autolesión no suicidas: una revisión sistemática. Psicología y Salud, 31(1), 51-59. https://doi.org/10.25009/pys.v31i1.2675.

RESUMEN

\begin{abstract}
Las conductas autolesivas implican hacerse daño sin la intención de llegar al suicidio, y son comportamientos que se consideran como un problema que va en aumento entre la población adolescente; de hecho, en México los datos estadísticos estiman que $10 \%$ de los adolescentes se autolesionan; no obstante, la investigación de los factores de riesgo contextuales que propician tales conductas se ha considerado inadecuada y limitada. Objetivo: Con la finalidad de recabar las variables y teorías actuales para abordar el problema, el propósito de esta revisión fue analizar la bibliografía especializada sobre los factores contextuales relacionados a las conductas de autolesión no suicida en adolescentes. Método: Se realizó una búsqueda exhaustiva en las bases de datos Scopus, EBSCO, Dialnet Plus y Scielo, considerando los artículos publicados de enero de 2015 a abril de 2019, y empleando las palabras clave self-injury, self-harm, adolescents, teenagers, risk factors, autolesión, adolescentes y factores de riesgo, ubicadas tanto en el título como en el resumen. Se seleccionaron diez trabajos que cumplieron los criterios de inclusión en la base bibliográfica. Resultados: Entre las variables estudiadas en los distintos artículos, relacionadas con la familia se encontraron como significativas la muerte de los padres, un historial de abuso y los conflictos familiares, mientras que en las vinculadas a la escuela la variable significativa fue la victimización en el acoso escolar.
\end{abstract}

Palabras clave: Conductas autolesivas; Factores de riesgo; Adolescente.

\begin{abstract}
Self-harm behaviors involve doing harm to oneself without the intention of reaching suicide. These are behaviors considered as an increasing problem among adolescent populations. In Mexico, statistical data estimate that 10\% of adolescents harm themselves. However, research on contextual risk factors on self-harm behaviors has been considered inadequate and limited. Objective: In order to gather current variables and theoretical explanations to address the problem, the purpose of this review was to analyze the research literature on contextual factors related to non-suicidal self-harm behaviors in adolescents. Method: An exhaustive search was carried out in the following databases: Scopus, EBSCO, Dialnet Plus and SciELO, including articles published from January 2015 to April 2019. The keywords self-injury, self-harm, adolescents, teenagers, risk factors, selfharm, adolescents, and factores de riesgo, were located in the title and summary. Ten papers that met the criteria for inclusion in the bibliographic database were selected. Results: The main variables significantly documented in the articles as related to family life were: parental death, history of abuse, and family conflicts. In relation to the school the most significant variables was victimization through bullying
\end{abstract}

Keywords: Self-harm behaviors; Risk factors; Adolescents.

\footnotetext{
${ }^{1}$ Centro de Investigación en Alimentación y Desarrollo A.C., Carretera Gustavo Enrique Astiazarán Rosas Núm. 46, Col. La Victoria, 83304 Hermosillo, Son., México, tel. (662)848-16-64, correos electrónicos: kareng_18@hotmail.com, avera@ciad.mx y daniel_fregoso14@hotmail.com. Artículo recibido el 12 de noviembre de 2019 y aceptado el 7 de abril de 2020.
} 
$\mathrm{L}$ os comportamientos autolesivos son aquellos que implican provocarse lesiones deliberadas y directas en la superficie corporal sin la intención de llegar al suicidio; se consideran socialmente inaceptables (Chapman, Gratz y Brown 2006; Chartrand, Bhaskaran, Sareen, Katz y Bolton, 2015; Lewis y Heath, 2015) y se realizan de forma repetitiva (Nicolai, Wielgus y Mezulis, 2016). Entre los métodos comunes de autolesión se encuentra el cortarse, quemarse y golpearse (Meza, Owens y Hinshaw, 2016), los que se consideran como alternativas para manejar situaciones o emociones intolerables (Santos, 2011).

A pesar de que las autolesiones son comportamientos que se han estudiado principalmente en muestras clínicas, constituyen un problema que ha aumentado considerablemente en la población general (Gratz, 2006; DeRiggi, Moumne y Heath, 2016), pero sobre todo entre los adolescentes (Nicolai et al., 2016; Ougrin, Banarsee, Dunn-Toroosian y Majeed, 2011).

Obtener datos certeros de las personas que se autolesionan de manera continua es una tarea ardua. Santos (2011) apunta que tal dificultad puede deberse a que es común el que las personas que lo hacen lo mantengan en secreto durante un tiempo prolongado; por otro lado, la definición de conducta autolesiva varía, lo que crea confusión, y por último los instrumentos de medición no son similares en las diversas investigaciones. Pese a ello, algunos estudios brindan informes estadísticos sólidos como los encontrados por Muehlenkamp y Gutiérrez (2007), que indican que la prevalencia de dicha conducta a lo largo de la vida va de 13 a $23.2 \%$. En México, los datos estadísticos estiman que $10 \%$ de los adolescentes se autolesionan (Nicolai et al., 2016), y asimismo que la prevalencia es de $17.1 \%$ en relación al periodo de tiempo, con la definición de cinco eventos en un año (Albores et al., 2014).

Los estudios sobre el comportamiento autolesivo han examinado los factores de riesgo que llevan a los individuos a realizar conductas de este tipo; sin embargo, se ha considerado que los mismos no han sido adecuados y que la atención que se le ha dado a los factores contextuales es limitada (Gratz, 2006; Santos, 2011).

Debido a que los adolescentes pasan una considerable parte de su tiempo en distintos contextos sociales vinculados a sus procesos de identidad y pertenencia, las variables sociales a las que se exponen influyen de un modo particular en su salud mental, por lo que el estudio de los contextos es fundamental para comprender los cambios en la tasa de las referidas conductas (Landstedt y Gillander, 2010). Entre los factores contextuales que se han estudiado y reconocido como relevantes en el problema de la autolesión no suicida se encuentra el ambiente familiar en la forma de escaso apego, conflictos frecuentes, relaciones familiares pobres (Obando, Trujillo y Prada, 2018) y la propia estructura familiar (Landstedt y Gillander, 2010). También hay evidencia de que la victimización escolar y otros tipos de violencia, como la física, la sexual y el acoso, son factores contextuales de riesgo que propician la ocurrencia de conductas de autolesión en los adolescentes, pero se necesitan más investigaciones acerca de estos y otros elementos (Landstedt y Gillander, 2010).

Los artículos que dan cuenta de los factores contextuales suelen centrar su atención en el abuso sexual, pero omiten otras variables de este tipo; sin embargo, el abuso sexual no es el único componente que se correlaciona con las autolesiones no suicidas (Gratz, Conrad y Roemer, 2002), y al ser la adolescencia una etapa de cambios en la que el individuo debe aprender estrategias que pueda usar de forma efectiva al alcanzar la adultez, el estudio de los factores relacionados con el mencionado comportamiento es fundamental (Castro y Kirchner, 2018).

A fin de recabar las variables y teorías actuales para abordar el citado problema, el propósito de la presente revisión fue examinar los artículos que analizan los factores contextuales relacionados con las conductas de autolesión no suicida en adolescentes.

\section{MÉTODO}

\section{Procedimiento}

Para tal propósito, se llevó a cabo una búsqueda exhaustiva de estudios publicados durante el periodo de enero de 2015 a abril de 2019 en las bases de datos Scopus, EBSCO, Dialnet Plus y SciELO y otra información personal y directa, al considerarse 
que el referido problema en Latinoamérica surge de un contexto empobrecido y de un sistema público de educación carente de recursos materiales, así como de tiempo y capacitación suficientes.

Los términos de búsqueda se dividieron en tres grupos, utilizando para ello las palabras en inglés "self-injury or self-harm", "adolescents or teenager" y "risk factors", y en español los términos "autolesión", "adolescentes" y "factores de riesgo", empleando los operadores booleanos $A N D$ y $O R$. Dichos términos se buscaron como parte del resumen o abstract y de las palabras clave en las tres bases de datos; sin embargo, al no existir estos términos en el tesauro de EBSCO, se emplearon las siguientes sinonimias: "self-injurious behavior", "adolescence" y "teenagers". Del mismo modo, se efectuó una búsqueda manual entre las referencias de los documentos que cumplían con los criterios de selección para encontrar posibles investigaciones que no hubiesen sido detectadas en la búsqueda en las bases de datos.

Se tomaron los siguientes criterios de inclusión: a) que los trabajos abordaran las autolesiones no suicidas, por lo que se descartaron los artículos que hablaran de las autolesiones como factor de riesgo para el intento de suicidio o el sui- cidio consumado; b) que la muestra utilizada estuviera constituida por adolescentes; c) que los artículos brindaran información sobre los factores contextuales relacionados con los comportamientos de autolesión, y d) que estuvieran publicados en revistas vinculadas a las bases bibliográficas.

La búsqueda inicial arrojó 28 resultados en la base de datos EBSCO, de los cuales se recuperaron cuatro; en Scopus se encontraron 16 resultados, de los que se seleccionaron cuatro, y Dialnet PLUS arrojó ocho resultados, ninguno de los cuales entró en esta revisión. Salvo en Dialnet PLUS, donde la búsqueda en español arrojó dos artículos de los ocho totales, y SciELO, en que se hallaron tres artículos, de los cuales ninguno cumplió con los criterios, todos los encontrados lo fueron a través de los conceptos empleados en idioma inglés. Mediante una búsqueda manual se encontraron dos documentos más. Así, se recuperaron diez artículos en total, los que fueron revisados.

La recolección de datos se llevó a cabo con base en los lineamientos de la metodología Elementos de informes preferidos para la revisión sistemática y el metanálisis (PRISMA) (Urrútia y Bonfill, 2010). El proceso de revisión sistemática se presenta en la Figura 1.

Figura 1. Diagrama de flujo de proceso de exclusión e inclusión.

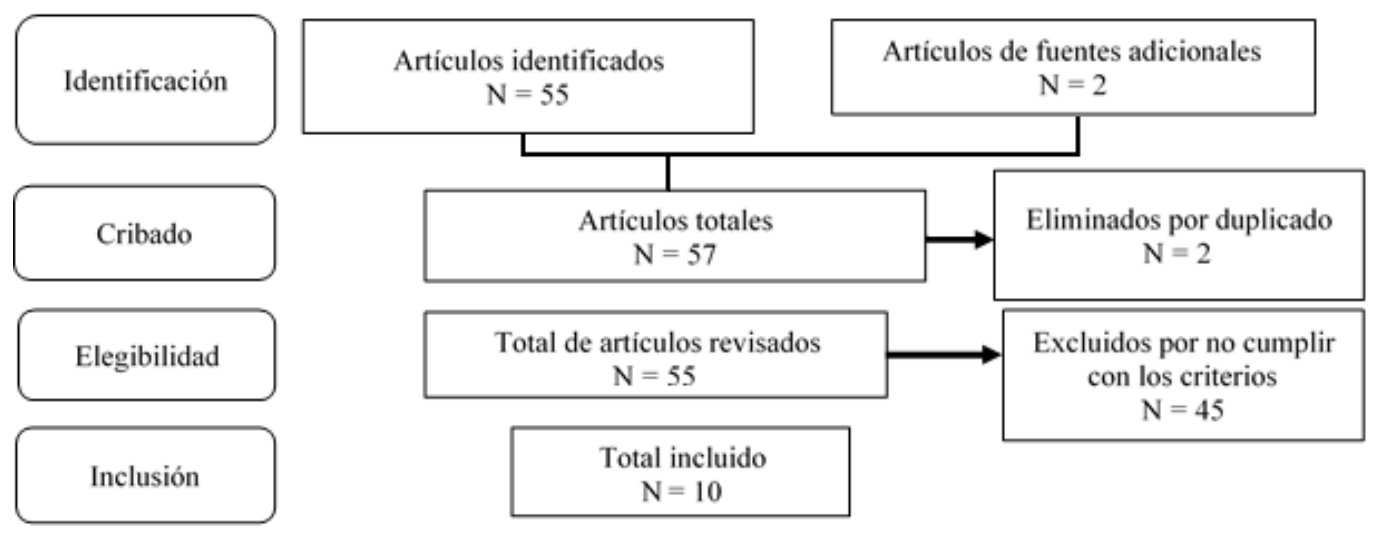

\section{RESULTADOS}

En los estudios de autolesión en adolescentes se han podido apreciar dos grupos de población: los que forman parte de la población general y los que constituyen una muestra clínica. Con el propósito de identificar diferencias entre ambos tipos de muestras, se abordan aquí como dos grupos separados.

Se presentan primeramente los artículos en los que la muestra utilizada pertenecía a la población general. Entre los estudios sobre la autolesión en adolescentes se han reportado hallazgos relacionados con los factores de riesgo contextua- 
les, como los encontrados en la base de datos de Scopus; en estos descubrimientos se encuentran los de Giletta et al. (2015), quienes a través de un modelo de trayectoria analizan las variables de autolesión, depresión, apoyo de amigos, victimización entre pares y tipo de amistad en una muestra de 565 adolescentes chinos de décimo grado, utilizando para ello un diseño de investigación longitudinal de dos años con tres meses de duración, con corte explicativo. En ese trabajo, que emplea un modelo de trayectoria conocido en inglés como path analysis, se halló que la victimización entre pares es un poderoso antecedente para que se desarrollen conductas de autolesión no suicida.

Kang et al. (2018) estudiaron la relación entre el maltrato infantil y la autolesión no suicida, mediada por la tolerancia a la angustia, en una muestra de 3,555 adolescentes de China con edades de entre 12 y 19 años. Se hicieron análisis de chi cuadrada y correlaciones de Pearson, y además diversos análisis de grupo múltiple siguiendo tres pasos. Los resultados mostraron que solamente el abuso emocional estaba indirectamente relacionado con la autolesión a través de la intolerancia a la angustia, toda vez que en las familias que abusan emocionalmente los padres descuidan e invalidan las emociones de los hijos, mientras que en las familias cálidas, tolerantes y armoniosas se responde empáticamente hacia las emociones de los mismos, lo que subraya la importancia del ambiente familiar en el desarrollo del adolescente.

Wang, You, Lin, Xu y Leung (2016) abordaron el estudio de varios factores de riesgo con el propósito de identificar los subgrupos heterogéneos de adolescentes con distintas trayectorias de comportamientos autolesivos, así como los factores de riesgo personales y contextuales en la trayectoria de las autolesiones no suicidas. Tales factores fueron la depresión, la ansiedad, la autocrítica, las relaciones inestables y la crítica de los padres. La muestra se constituyó por 5,423 adolescentes que se encontraban estudiando en una de las ocho escuelas secundarias seleccionadas de Hong Kong. El diseño del estudio fue transversal, llevándose a cabo una prueba $t$ para muestras independientes mediante un análisis de crecimiento de clases latente para determinar si había diferentes trayectorias de desarrollo en la población. Los resultados indicaron que los adolescentes que informaron de factores de riesgo tanto personales como contextuales fueron significativamente más propensos a desarrollar las mencionadas conductas de autolesión no suicida, pues se encontró que niveles altos de depresión, ansiedad, relaciones inestables y críticas de los padres se asociaron significativamente a un incremento de la posibilidad de experimentar autolesiones; además, entre los subgrupos formados, el de alta fluctuación de conductas de autolesión mantuvo un nivel persistentemente elevado de esos comportamientos.

Otro estudio que analizó los factores relacionados con la familia fue el realizado por $\mathrm{Hu}$, Li, Glauert y Taylor (2017), cuyo objetivo fue identificar factores de riesgo que predijeran el ingreso hospitalario por conductas autolesivas, para lo cual consideraron las variables de desventaja social temprana, trastornos de salud mental de los padres y muerte parental. Los autores utilizaron un diseño transversal explicativo con la información obtenida a través de una vinculación de datos creados por la base de datos de registro de nacimiento de Australia, país en que las agencias gubernamentales recogen de manera rutinaria los datos relacionados con la salud. La vinculación de los datos proporciona la información necesaria para vincular los registros que pertenecen a una misma persona. Se seleccionaron los casos de adolescentes de entre 10 y 19 años, hospitalizados por algún tipo de autolesión, los cuales sumaron un total de 7,151 individuos. A partir de una regresión logística, se encontró que invariablemente la muerte del padre o de la madre tenía efectos significativos como factor de riesgo para la autolesión no suicida, aunque en el caso de la muerte de la madre el efecto era mucho mayor.

Garisch y Wilson (2015) evaluaron la prevalencia y la correlación de las conductas de autolesión no suicida con las variables personales y contextuales, como depresión, ansiedad, autoestima, uso adaptativo de las emociones, preocupación sexual, impulsividad, acoso escolar y abuso. El estudio transversal causal se llevó a cabo en una muestra de adolescentes de Nueva Zelanda. Se realizaron correlaciones de Pearson para valorar la relación entre autolesión y cada una de las variables y paneles de regresión cruzada para evaluar la relación de cada variable a través del tiempo. En relación con los factores contextuales, los 
resultados indicaron que las autolesiones se asociaban con un historial considerable de abuso, y además que el acoso escolar puede ser un predictor distal de tal conducta.

Por otro lado, un trabajo de Stanford, Jones y Hudson (2017) tuvo como objetivo estudiar la superposición de los factores de riesgo en los comportamientos autolesivos de estudiantes de secundaria australianos, entre los que se seleccionaron depresión, ansiedad, autoestima, dificultades conductuales y de atención, estrategias de afrontamiento y satisfacción con la vida, además de variables contextuales como relaciones de apoyo, acoso escolar y prácticas religiosas, para así conformar perfiles psicológicos que hicieran posible identificar a los adolescentes más propensos a autolesionarse. Mediante un análisis de clúster no jerárquico, se generaron seis grupos con diferentes perfiles psicológicos. Los resultados obtenidos reafirman los de estudios anteriores, pues se encontró que ser víctima de acoso escolar es un factor de riesgo importante, especialmente en personas con perfiles impulsivos.

El trabajo de Heerde et al. (2015) tuvo como objetivo analizar los factores protectores y de riesgo relacionados a la incidencia y la persistencia de las conductas autolesivas mediante un estudio longitudinal. La muestra estuvo conformada por adolescentes entre 13 y 15 años estudiando el séptimo y noveno grado en el estado de las ciudades de Washington (Estados Unidos) y Victoria (Australia). Para comparar las medias y frecuencias de los factores de riesgo entre las muestras, se efectuaron pruebas $t$ de Student, análisis de chi cuadrada y un modelo de regresión logística multivariante para investigar los mencionados factores protectores y de riesgo. Los componentes analizados fueron, a saber: síntomas de depresión, comportamientos antisociales, impulsividad, uso de alcohol, victimización en acoso escolar, control parental y conflictos familiares. Los resultados mostraron que todas las variables tuvieron correlaciones significativas de los comportamientos autolesivos, y además que los estudiantes de Victoria alcanzaron niveles más altos de riesgo de victimización por acoso escolar, impulsividad, manejo familiar deficiente y consumo de tabaco, que los estudiantes de Washington, siendo también los que presentaron una mayor incidencia y persistencia de tales conductas.

Entre los estudios en los que la población estaba conformada por una muestra clínica, se encontraron los siguientes factores contextuales asociados a la autolesión.

Por ejemplo, el maltrato infantil fue investigado por Titelius et al. (2017) examinando la relación entre dicho maltrato y las autolesiones no suicidas, así como la variable de regulación emocional. La muestra incluyó a 53 adolescentes con edades de entre 12 y 18 años que formaban parte de un programa de un hospital psiquiátrico infantil de Estados Unidos. Los análisis realizados fueron correlaciones de Pearson y path analysis. Los resultados mostraron que el maltrato físico y emocional se asociaba de manera significativa con las autolesiones, no así el abuso sexual.

Del mismo modo, Peh et al. (2017) estudiaron el maltrato infantil en relación con la desregulación emocional. En su estudio participaron 108 adolescentes recluidos en un hospital psiquiátrico de Singapur, con una media de edad de 17 años. Se analizaron las variables de maltrato infantil, autolesiones, desregulación emocional y depresión, y los resultados indicaron que la desregulación emocional puede ser un mecanismo que vincula la exposición al maltrato y la conducta autolesiva en los adolescentes, por lo que se considera que esta última funciona como una conducta que revela la incapacidad para regular las emociones como resultado del maltrato sufrido en la infancia. Los resultados se obtuvieron asimismo utilizando un análisis de correlación y un path analysis.

Por último, Klassen, Hamza y Stewart (2017) estudiaron una muestra de 541 adolescentes con acceso a servicios de salud mental en veinte comunidades de Ontario, abordando las variables de conducta autolesiva, uso de sustancias, depresión, agresión, preocupación del cuidador y violencia en el barrio. Mediante una entrevista semiestructurada, se obtuvo información cualitativa y cuantitativa, y los datos se analizaron mediante la regresión logística multinominal. Los resultados revelaron que las mujeres que manifestaban síntomas depresivos intensificados y sufrían violencia en su barrio corrían un mayor riesgo de autolesionarse. 


\section{DISCUSIÓN}

La presente revisión permitió conocer algunos aspectos importantes del estado actual de la investigación de los factores de riesgo relacionados con el desarrollo y la prevalencia de conductas de autolesión en adolescentes. Los resultados obtenidos hacen posible constatar que las muestras clínicas han recibido una mayor atención en el estudio de los factores contextuales, lo que coincide con lo encontrado por Gratz (2006) y DeRiggi et al. (2016), quienes afirman que el análisis de las mencionadas conductas se ha centrado en poblaciones clínicas. Lo anterior supone un problema en la investigación del fenómeno, ya que la mayoría de datos de que se dispone están focalizados particularmente en esas poblaciones, lo que dificulta el estudio de un problema que va en aumento entre la población adolescente escolarizada (Ulloa, Contreras, Paniagua y Victoria, 2013).

Por otro lado, ninguno de los artículos revisados se llevó a cabo en países latinoamericanos, lo que respalda las afirmaciones de Castro et al. (2016), quienes señalan que el comportamiento autolesivo no suicida es un problema que no ha recibido la atención necesaria en la investigación, lo que incluye a México. En efecto, no hay trabajos que estudien cuestiones tales como los instrumentos confiables para detección de esas conductas y las variables asociadas al fenómeno, especialmente en la población general.

Entre los factores contextuales que han recibido aún menos atención se encuentran los relacionados con la comunidad; de hecho, solamente se le abordó en uno de los artículos revisados, al contrario de los factores relacionados con la familia y a la escuela, analizados con mayor frecuencia y con resultados significativos. Es posible que lo anterior se deba a que la familia es un ambiente esencial en el desarrollo en virtud de que es el entorno primario de interacción (Castro et al., 2016) y provee la formación necesaria para que el menor forme parte de la sociedad (Curtis, 2015; Klemera, Brooks, Chester, Magnusson y Spencer, 2016). A pesar de que las conductas de autolesión han mostrado estar generadas por variables sociales, la falta de interés por estudiar la comunidad, así como los factores de riesgo y protección asociados a la colonia o el barrio, puede deberse a que no se ha encontrado evidencia de que las habilidades sociales que permiten obtener la aceptación y la pertenencia al grupo sean un factor protector para evitar aquellas entre los adolescentes (Obando et al., 2018).

Considerando la información plasmada en los resultados de los artículos analizados, es posible afirmar que la relación con los padres y la interacción familiar son factores importantes para desarrollar comportamientos de autolesión, de manera que investigar tales factores hará posible una prevención e intervención más efectivas sobre el mencionado fenómeno (Adrian et al., 2018; Berndt, 1979; Ren et al., 2018).

Otro factor que se ha aborda con particular interés en las investigaciones revisadas es el relacionado con el acoso escolar, y en particular con la victimización; lo que ocurre dentro de las instituciones educativas no solo debiera estar enfocado a la adquisición de conocimientos académicos, sino también a los procesos de socialización que tienen lugar en la escuela, pues es un espacio de socialización en el que los niños y adolescentes pasan una parte considerable del tiempo interactuando con sus pares y profesores (Gallegos, Casapia y Rivera, 2018; Madjar et al., 2017). Así, constituye un contexto vital para obtener información sobre el comportamiento autolesivo.

Las formas de autolesión que los adolescentes presentan en América Latina se asemejan a las que se encuentran en América del Norte y Europa; sin embargo, factores como la pobreza y la inseguridad se han subestimado en aquella, pues es posible que la presencia de estas circunstancias sociales aumente el riesgo de desarrollar tales conductas (Hilton, 2016; Klonsky y Lewis, 2014).

De acuerdo al objetivo planteado, los factores estudiados para explicar las conductas de autolesión en cada uno de los contextos fueron los siguientes: en el caso de la familia, se detectaron el maltrato infantil, los trastornos de salud mental de los padres (Wang et al., 2016), la muerte parental, un historial de abuso, el control parental y los conflictos familiares (Stanford et al., 2018). En el escenario escolar, las variables analizadas fueron el apoyo familiar, el tipo de amistad (Giletta et al., 2015) y la victimización debida al acoso escolar (Giletta et al., 2015; Hu et al., 2017; Garisch y Wilson, 2015; Stanford et al., 2018), resultando 
significativa solamente esta última (Giletta et al., 2015; Hu et al., 2017; Garisch y Wilson, 2015; Stanford et al., 2018).

A pesar de que en la investigación de los factores relacionados a los comportamientos autolesivos se han abordado diversos entornos del adolescente en la investigación, aún el público en general no comprende bien la problemática, por lo que un entendimiento más profundo del asunto mediante un consenso sobre la terminología empleada en las publicaciones (Silva y Van Camp, 2014) y la construcción de estudios empíricos y teóricos que permitan reconocer otros factores de riesgo y variables que se asocian a la aparición, evolución y mantenimiento de las conductas de autolesión (Obando et al., 2018) podrían ser un considerable apoyo para prevenir e intervenir en las poblaciones de riesgo (Silva y Van Camp (2014).

En definitiva, estudiar los multicitados comportamientos y los factores de riesgo implicados hará posible comprender el sentido de estas conductas, así como diseñar estrategias y seleccionar tratamientos apropiados para cada individuo (Ougrin et al., 2011). Por consiguiente, el estudio de las autolesiones no suicidas podrá proporcionar un fundamento teórico en lo que a la ciencia psicológica respecta, explorando, describiendo, definiendo y delimitando la conducta misma, sus causas y efectos, para esclarecer y proporcionar guías para la toma de decisiones relacionadas con los diagnósticos, así como permitir el desarrollo del estado del arte y técnicas de investigación novedosas (Ordóñez, 2018), ya que, según se ha visto en esta revisión, la mayor parte de literatura científica proviene de estudios clínicos y de otros que aportan información del fenómeno, pero que dejan de lado muchos factores contextuales y los rasgos de personalidad.

Aun revisando el problema de autolesión en bases de datos de calidad, generalmente son escasas las investigaciones científicas (55), pero solo 10 intentan extrapolar el problema para entender los factores que funcionen como posibles causas. Por ello, los hallazgos de esta revisión pretenden dar pie y motivar el estudio más acucioso del fenómeno en cuestión al tratarlo como uno de tipo multifactorial y multicausal, en el que desempeñan un importante papel elementos contextuales tales como la compleja socialización en ambientes académicos, las configuraciones e interacciones familiares, e incluso los probables factores de riesgo y de protección asociados al uso del espacio público.

\section{REFERENCIAS}

Adrian, M., Berk, M., Korslund, K., Whitlock, K., McCauley, E. y Linehan, M. (2018). Parental validation and invalidation predict adolescent self-harm. Professional Psychology: Research and practice, 49(4), 274-281. Doi: 10.1037/pro0000200.

Albores, L., Méndez, J., García, A., Delgadillo, D., Chávez, C. y Martínez, O. (2014). Autolesiones sin intención suicida en una muestra de niños y adolescentes en la Ciudad de México. Actas Españolas de Psiquiatría, 42(4), 159-168.

Berndt, T. (1979). Developmental changes in conformity to peers and parents. Developmental Psychology, 15, 608-616. Doi: 10.1037/0012-1649.15.6.608.

Castro, E., Benjet, C., Juárez, F., Jurado, S., Gómez, M. y Valencia, A. (2016). Adaptación y propiedades psicométricas del Inventory of Statemnts About Self-injury en estudiantes mexicanos. Actas de Investigación Psicológica, 6, $2544-2551$. Doi: 10.1016/j.aipprr.2016.08.004.

Castro, K. y Kirchner, T. (2018). Coping and psychopathological profile in nonsuicidal-self-injures in Chilean adolescents. Journal of Clinical Psychology, 74(1), 147-160. Doi: 10.1002/jclp.22493.

Chapman, A., Gratz, K. y Brown, M. (2006). Solving the puzzle of deliberate self-harm: The experiential avoidance model. Behaviour Research and Therapy, 44, 371-394. Doi: 10.1016/j.brat.2005.03.005.

Chartrand, H., Bhaskaran, J., Sareen, J., Katz, L. y Bolton, J. (2015). Correlates of nonsuicidal self-injury and suicide attempts among tertiary care, emergency department patients. Canadian Journal of Psychiatry, 60(6), 276-283. Doi: $10.1177 / 070674371506000606$.

Curtis, A. (2015). Defining adolescence. Journal of Adolescent and Family Health, 7, Article 2.

DeRiggi, M., Moumne, S. y Heath, N. (2016). Non-suicidal self-injury in our schools: A review and research-informed guidelines for school mental health professionals. Canadian Journal of School Psychology, 32(2), 122-143. Doi: $10.1177 / 0829573516645563$. 
Gallegos, M., Casapia, Y. y Rivera, R. (2018). Estilos de personalidad y autolesiones en adolescentes de Arequipa. Interacciones, 4(2), 143-151. Doi: 10.24016/2018.v4n2.106.

Garisch, J. y Wilson, M. (2015). Prevalence, correlates, and prospective predictors of non-suicidal self-injury among New Zealand adolescents: cross-sectional and longitudinal survey data. Child and Adolescent Psychiatry and Mental Health, 9(1), 1-11. Doi: 10.1186/s13034-015-0055-6.

Giletta, M., Abela, J., Prinstein, M., Gibb, B., Barrocas, A. y Hankin, B. (2015). Trajectories of suicide ideation and nonsuicidal self-injury among adolescents in Mainland China: Peer predictors, joint development, and risk for suicide attempts. Journal of Consulting and Clinical Psychology, 83(2), 265-279. Doi: 10.1037/a0038652.

Gratz, K. (2006). Risk factors for and functions of deliberate self-harm: An empirical and conceptual review. Clinical Psychology: Science and Practice, 10(2), 192-205. Doi: 10.1093/clipsy.bpg022.

Gratz, K., Conrad, S. y Roemer, L. (2002). Risk factors for deliberate self-harm among college students. American Journal of Orthopsychiatry, 72(1), 128-140.

Heerde, J., Toumbourou, J., Hemphill, S., Herrenkohl, T., Patton, G. y Catalano, R. (2015). Incidence and course of adolescent deliberate self-harm in Victoria, Australia, and Washington State. Journal of Adolescent Health, 57(5), 537-544. Doi: 10.1016/j.jadohealth.2015.07.017.

Hilton, C. (2016). Unveiling self-harm behavior: What can social media site Twitter tell us about self-harm? A qualitative exploration. Journal of Clinical Nursing, 26, 1690-1740. Doi: 10.1111/jocn.13575.

$\mathrm{Hu}, \mathrm{N} ., \mathrm{Li}, \mathrm{J} ., \mathrm{Glauert,} \mathrm{R.} \mathrm{y} \mathrm{Taylor,} \mathrm{C.} \mathrm{(2017).} \mathrm{Influence} \mathrm{of} \mathrm{exposure} \mathrm{to} \mathrm{perinatal} \mathrm{risk} \mathrm{factors} \mathrm{and} \mathrm{parental} \mathrm{mental} \mathrm{health} \mathrm{related}$ hospital admission on adolescent deliberate self-harm risk. European Child \& Adolescent Psychiatry, 26 (7), $791-803$. Doi: 10.1007/s00787-017-0948-4.

Kang, N., Jiang, Y., Ren, Y., Gong, T., Liu, X., Leung, F. y You, J. (2018). Distress intolerance mediates the relationship between child maltreatment and nonsuicidal self-injury among Chinese adolescents: A three-wave longitudinal study. Journal of Youth and Adolescence, 47(10), 2220-2230. Doi: 10.1007/s10964-018-0877-7.

Klassen, J., Hamza, C. y Stewart, S. (2017). An examination of correlates for adolescent engagement in nonsuicidal self-injury, suicidal self-injury, and substance use. Journal of Research on Adolescence, 28(2), 342-353. Doi: 10.1111/jora.12333.

Klemera, E., Brooks, F., Chester, K., Magnusson, J. y Spencer, N. (2016). Self-harm in adolescence: protective health assets in the family, school and community. International Journal of Public Health, 62(6), 631-638. Doi: 10.1007/s00038-016-0900-2.

Klonsky, E. y Lewis, S. (2014). The Oxford Handbook of Suicide and Self-injury. New York: Oxford University Press.

Landstedt, E. y Gillander, K. (2010). Deliberate self-harm and associated factors in 17 year-old Swedish students. Scandinavian Journal of Public Health, 39(1), 17-25. Doi: 10.1177/1403494810382941.

Lewis, S.P. y Heath, N.L. (2015). Nonsuicidal self-injury among youth. The Journal of Pediatrics, 166(3), 526-530. Doi: 10.1016/j.jpeds.2014.11.062.

Madjar, N., Ben, S., Elia, R., Fellner, N., Rehavi, M., Rubin, S. y Shoval, G. (2017). Non-suicidal self-injury within the school context: Multilevel analysis of teacher's support and peer climate. European Psychiatry, 41, 95-101. Doi: 10.1016/j. eurpsy.2016.11.003.

Meza, J., Owens, E. y Hinshaw, S. (2016). Response inhibition, peer preference and victimization, and self-harm: Longitudinal associations in young adult women with and without ADHD. Journal of Abnormal Child Psychology, 44(2), 323-334. Doi: 10.1007/s10802-015-0036-5.

Muehlenkamp, J. y Gutierrez, P. (2007). Risk for suicide attempts among adolescents who engage in non-suicidal self-injury. Archives of Suicide Research, 11(1), 69-82. Doi: 10.1080/13811110600992902.

Nicolai, K., Wielgus, D. y Mezulis, A. (2016). Identifying risk for self-harm: Rumination and negative affectivity in the prospective prediction of nonsuicidal self-injury. Suicide and Life-threatening Behavior, 46(2), 223-233. Doi: 10.1111/sltb.12186.

Obando, D., Trujillo, Á. y Prada, M. (2018). Conducta autolesiva no suicida en adolescentes y su relación con factores personales y contextuales. Revista de Psicopatología Clínica, 23(3), 189-200. Doi: 10.5944/rppc.vol.23.num.3.2018.21278.

Ordóñez, G. (2018). Discriminación, pobreza y vulnerabilidad: los entresijos de la desigualdad social en México. Región y Sociedad, 30(71), 1-30. Doi: 10.22198/rys.2018.71.a377.

Ougrin, D., Banarsee, R., Dunn-Toroosian, V. y Majeed, A. (2011). Suicide survey in a London borough: primary care and public health perspectives. Journal Public Health Oxford England, 33(3), 385-391. Doi: 10.1093/pubmed/fdq094.

Peh, C., Shahwan, S., Fauziana, R., Mahesh, M., Sambasivam, R., Zhang, Y. y Subramaniam, M. (2017). Emotion dysregulation as a mechanism linking child maltreatment exposure and self-harm behaviors in adolescents. Child Abuse and Neglect, 67, 383-390. Doi: 10.1016/j.chiabu.2017.03.013.

Ren, Y., Lin, M., Liu, Y., Zhang, X., Wei, J., Hu, W. y You, J. (2018). The mediating role of coping strategy in the association between family functioning and nonsuicidal self-injury among Taiwanese adolescents. Journal of Clinical Psychology, 74, 1246-1257. Doi: 10.1002/jclp.22587.

Santos, D. (2011). Autolesión: Qué es y cómo ayudar. México: Edición de Autor.

Silva, L. y Van Camp, I. (2014). Non-suicidal self-injury in Latin America. Salud Mental, 37(2), 153-157. 
Stanford, S., Jones, M. y Hudson, J. (2017). Appreciating complexity in adolescent self-harm risk factors: psychological profiling in a longitudinal community sample. Journal of Youth and Adolescence, 47(5), 916-931. Doi: 10.1007/s10964-017-0721-5.

Titelius, E., Cook, E., Spas, J., Orchowski, L., Kivisto, K., O’Brien, K. y Semour, K. (2017). Emotional dysregulation mediates the relationship between child maltreatment and non-suicidal self-injury. Journal of Aggression, Maltreatment and Trauma, 27(3), 323-331. Doi: 10.1080/10926771.2017.1338814.

Ulloa, R., Contreras, C., Paniagua, K. y Victoria, G. (2013). Frecuencia de autolesiones y características clínicas asociadas en adolescentes que acudieron a un hospital psiquiátrico infantil. Salud Mental, 36(5), 417-420.

Urrútia, G. y Bonfill, X. (2010). Declaración PRISMA: Una propuesta para mejorar la publicación de revisiones sistemáticas y metaanálisis. Medicina Clínica, 132(11), 507-511. Doi: 10.1016/j.medcli.2010.01.015.

Wang, B., You, J., Lin, M., Xu, S. y Leung, F. (2016). Developmental trajectories of nonsuicidal self-injury in adolescence and intrapersonal/interpersonal risk factors. Journal of Research on Adolescence, 27(2), 392-406. Doi: 10.1111/jora.12273. 